\title{
Surgical Management of Large Mediastinal Masses-12 Years' Experience in Apollo Hospitals Dhaka
}

\author{
Zahangir $\mathrm{NM}^{1}$, Hasan $\mathrm{MK}^{2}$, Basak RK ${ }^{3}$, Meher $\mathrm{T}^{4}$, Alam $\mathrm{NS}^{5}$, Hossain $\mathrm{MN}^{6}$, Khan $\mathrm{A}^{7}$,
} Ahmed $\mathrm{N}^{8}$, Ahmed $\mathrm{S}^{9}$, Haider MZ ${ }^{10}$

\begin{abstract}
Objective: The aim of this study was to evaluate the role of surgical treatment for large mediastinal tumors. A retrospective study was done from 6.8.2005 to 11.5.2017. Total 39 patients were included in this study. Age range was from 7 months to 70 years. Male was 24 and female was 15. Background: Most of the tumours were thymoma (12 cases). Others were thymoma with myasthenia gravis in 3 cases, Thymic carcinoma 2 cases, Nodular sclerosing Hodgkin's lymphoma in 1 case, Schwannoma in 3 cases, Cystic mass in 5 cases, Schwannoma with cystic degeneration in 1 case, Fibromyxoma in 1 case, Dermoid in 4 cases, Teratoma in 3 cases, Fibrosing mediastinitis in thymic mass in 1 case, Lymphoid hyperplasia in 1 case, Malignant Mesenchymal tumour in 1 case, Myxoid Liposarcoma in 1 case. Method: Surgical exposure varies from sternotomy in 22 cases, to clamshell incision in 1 case, posterolateral thoracotomy in 14 cases and anterolateral thoracotomy in 2 cases. Superior Venacava was injured in 1 case, repaired successfully by $6 / 0$ prolene controlled by partial clamp. Some of the mediastinal mass needed careful dissection from lung, pleura, encircling distal aortic arch and proximal part of left subclavian artery, encircling left brachiocephalic vein. 1 case needed reconstruction of sternum with prolene mesh, marsupialization was done in 2 cases, adjacent pleura, fatty tissue, part of pericardium was removed in 5 cases. Lobectomy was needed in 1 case. In 1 case, recurrence of fibrosing mediastinitis needed steroid therapy. Extensive growth of the tumour which involved surrounding vital structures was decided not to proceed for excision in 2 cases. Results: Overall five-years survival rate was $94.87 \% .2$ patients of the series died due to disease process as they were cases of advanced malignant mediastinal mass. Conclusion: Surgical treatment for mediastinal mass specially involving surrounding structures though challenging, gives good outcome.
\end{abstract}

\section{Keywords}

Mediastinal masses, thymoma, schwannoma, fibromyxoma, dermoid, teratoma, myxoid liposarcoma

1. Associate Consultant, Dept. of Cardiovascular and Thoracic Surgery, Apollo Hospitals, Dhaka 2. Registrar, Dept. of Cardiovascular and Thoracic Surgery, Apollo Hospitals, Dhaka 3. Registrar, Dept. of Cardiovascular and Thoracic Surgery, Apollo Hospitals, Dhaka 4. Registrar, Cardiothoracic Surgery, Apollo Hospitals, Dhaka 5. Registrar, Dept. of Cardiovascular and Thoracic Anesthesia, Apollo Hospitals, Dhaka 6. Sr. Registrar, Dept. of Cardiovascular and Thoracic Anesthesia, Apollo Hospitals, Dhaka 7. Resident Medical Officer, Dept. of Cardiovascular and Thoracic Surgery, Apollo Hospitals, Dhaka 8. Consultant, Dept. of Cardiothoracic Anesthesia, Apollo Hospitals, Dhaka 9. Consultant, Dept. of Cardiovascular and Thoracic Surgery, Apollo Hospitals, Dhaka 10. Consultant, Dept. of Cardiovascular and Thoracic Surgery, Apollo Hospitals, Dhaka 


\section{Introduction}

Mediastinal masses represent a vast group of tumours and pseudo-tumours which can involve the various compartments of the mediastinum. ${ }^{1}$ The most common mediastinal masses are neurogenic tumors (20\% of mediastinal tumors), usually found in the posterior mediastinum, followed by thymoma (15-20\%) located in the anterior mediastinum. Surgical removal of mediastinal masses usually gives good result.

\section{Materials and Methods}

A retrospective study was done from 06/08/2005 to $11 / 05 / 2017$. Total 39 patients were included in this study. Age range was from 30 years to 70 years. Male was 24 and female was 15. Most of the tumours were thymoma (12 cases), thymoma was associated with myasthenia gravis in 3 cases, Thymic carcinoma in 2 cases, Nodular sclerosing Hodgkin's lymphoma in 1 case, schwannoma in 3 cases, cystic mass in 5 cases, schwannoma with cystic degeneration in 1 case, fibromyxoma in 1 case, dermoid in 4 cases, teratoma in 3 cases, Fibrosing mediastinitis in thymic mass in 1 case, lymphoid hyperplasia in 1 case, malignant mesenchymal tumour in 1 case, myxoid liposarcoma in 1 case.

Symptomatically most common symptom was cough and chest pain. Second most common symptom was breathlessness and heaviness over the anterior chest wall. Other complains was intermittent fever, stabbing type of chest pain, difficulty in swallowing, dropping of upper eye lid, weight loss, anorexia, low grade fever, oral ulcer, swelling over lower part of the sternum.
Double lumen endotracheal tube intubation was used in all patients and fiber optic bronchoscope was used. Surgical exposures vary from sternotomy (22 cases) to clamshell incision in 1 case to posterolateral thoracotomy in 14 cases and anterolateral thoracotomy in 2 cases. Superior Venacava was injured in 1 case, repaired successfully by $6 / 0$ prolene controlled by partial clamp. Some of the mediastinal mass needed careful dissection from lung, pleura, encircling distal aortic arch and proximal part of left subclavian artery, encircling left brachiocephalic vein.

1 case needed reconstruction of sternum with prolene mesh, marsupialization was done in 2 cases, adjacent pleura, fatty tissue, part of pericardium was removed in 5 cases. Lobectomy was needed in 1 case. Extensive growth of the tumour which involve surrounding vital structures was decided not to proceed for excision in 2 cases.

Different type of presentation was found during operation. Large cystic mediastinal tumour was adherent with lower part of left lung and pericardium, separated carefully. Bronchial cyst was found tightly adherent to surrounding structures, excised the roof and suck out the chocolate coloured thick secretion, marsupialization of excised border was done- - kept the mouth wide open.

Thymic carcinoma was hard like stone adherent with the sternum, pericardium, left and right pleura, upper mediastinal surface of right lung, also encircling the left brachiocephalic vein, left lobe of the mass was removed totally along with 
was also removed as much as possible. Histopathologically, it was found to be epidermoid non-keratinizing carcinoma.

Thymoma with Myasthenia Gravis - total thymectomy along with removal of mediastinal fat, adjacent pleura, fatty tissue and small part of pericardium was removed.

Large fibrosarcoma $(8.5 \mathrm{~kg})$ - malignant mesenchymal tumour, excision of 1-4 ribs posteriorly, partial removal of left scapula and partly left pleura was excised.

Myxoid liposarcoma - adherent to great vessels pleura, chest walls, lungs, diaphragm and heart. Frozen section biopsy showed malignancy. Sample of tissue was then sent for histopathology. Clamshell - curvilinear bilateral

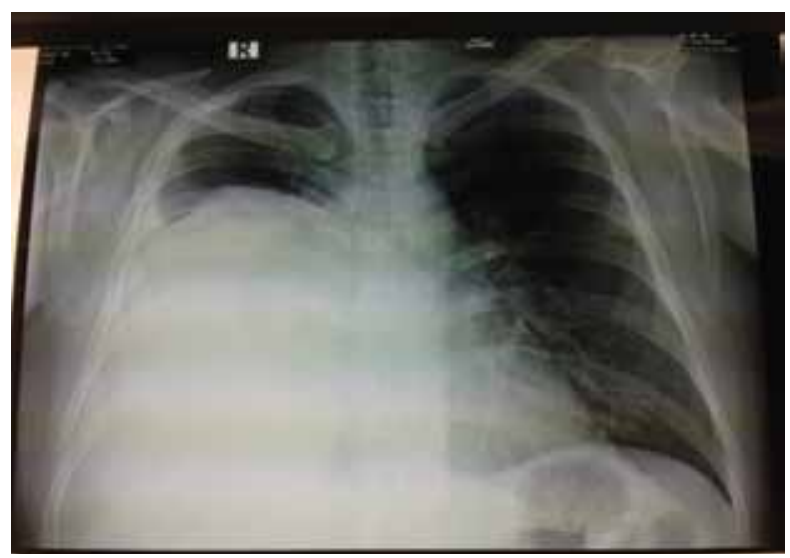

Fig. 1: Posterior mediastinal myxoid liposarcoma

submammary incision was performed extending from one mid axillary line to the opposite across the anterior aspect of chest, histopathologically found mature cystic teratoma-30x10x6 cm.

Spindle cell thymoma (Fig. 6) exposed by anterolateral thoracotomy in 70 years old gentleman, very large tumour between upper and middle middle lobe of right lung and in front of hilum of right lung, was firmly adherent to Superior Venacava - small tear in Superior Venacava repaired by $6 / 0$ prolene. Right mediastinal mass--FNAC showed benign cystic teratoma. During operation found inoperable-- frozen section biopsy showed malignancy-further surgical procedure abandoned as it was densely adherent to lung, pericardium and diaphragm.

\section{Results}

Overall five-years survival rate was 94.87\%. In 1 case recurrence of fibrosing mediastinitis needed steroid therapy. 2 cases were inoperable and died due to diseases process as they were advanced malignant mediastinal mass (fibrosarcoma and liposarcoma).

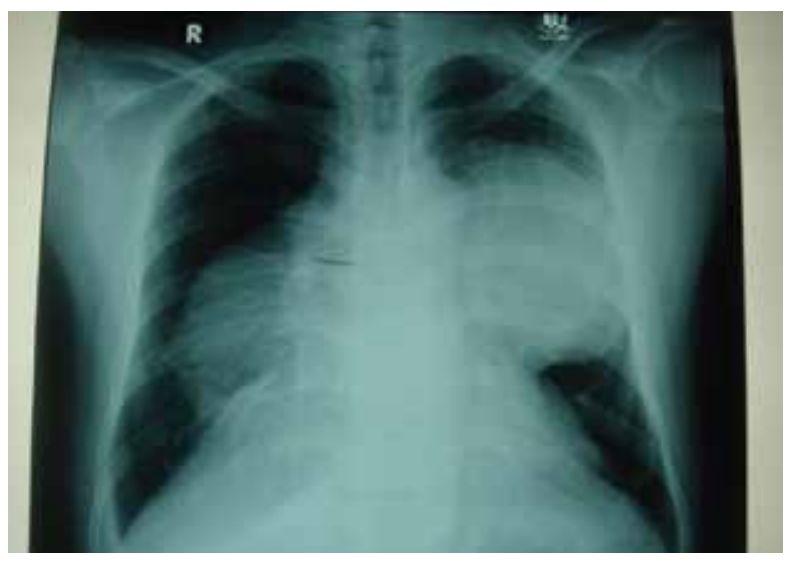

Fig. 2: Huge mediastinal tumour ---teratoma compressing both lungs and heart below

\section{Discussion}

Tumors of the mediastinum represent a wide diversity of disease states. The location and composition of a mass is vital for differential diagnosis. The most common causes of an anterior mediastinal mass include the following: thymoma, teratoma, thyroid disease and 


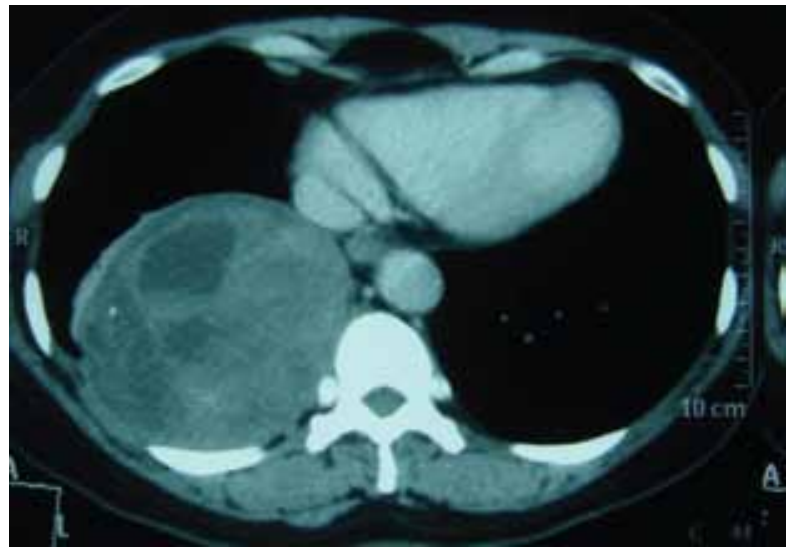

Fig. 3: CT scan looks to be a complex hydatid cyst in posterobasal segment of right lower lobe

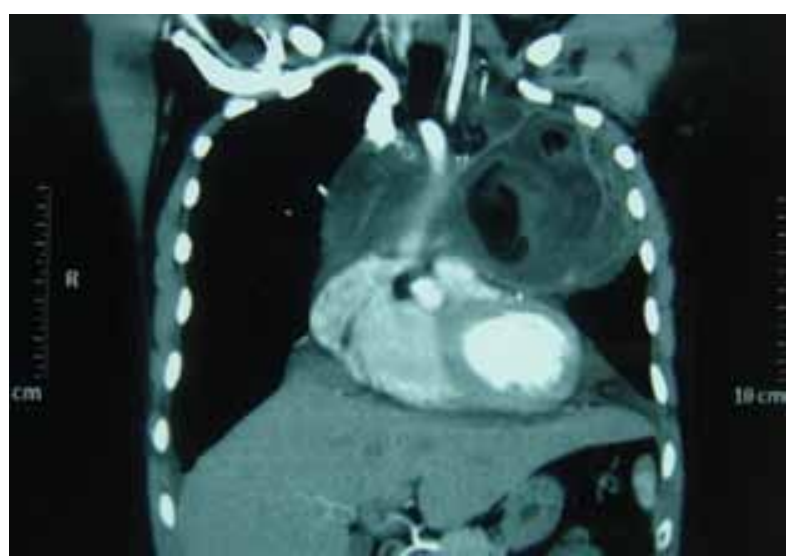

Fig. 5: CT scan-- mature cystic teratoma heart is pushed down

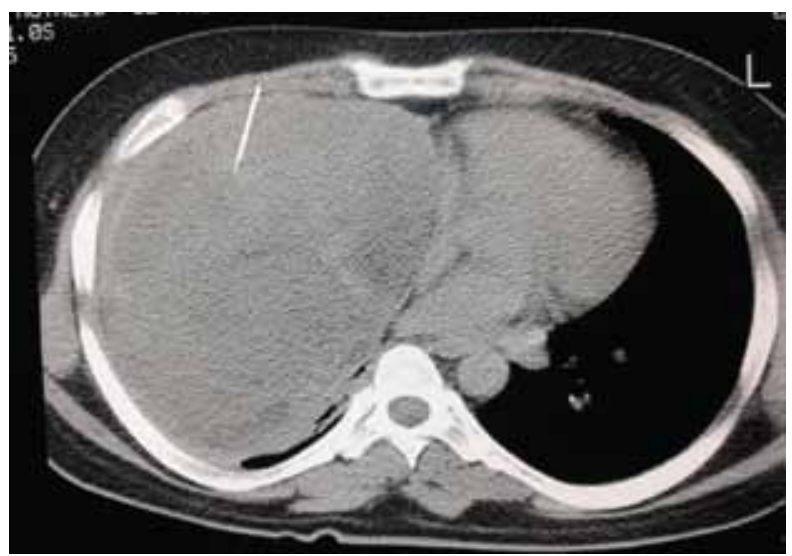

Fig. 7: 30 years gentle man: FNAC revealed Dermoid cyst, but peroperative frozen section biopsy showed myxoid liposarcoma

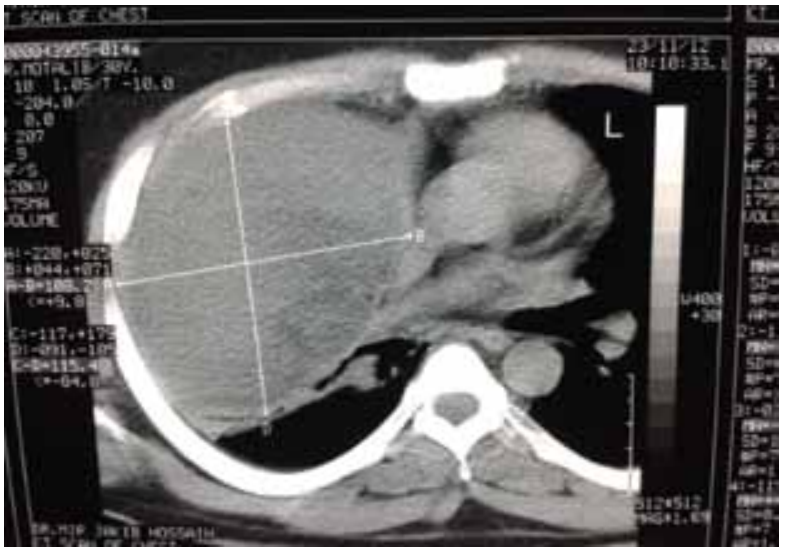

Fig. 4: Myxoid liposarcoma-inoperable

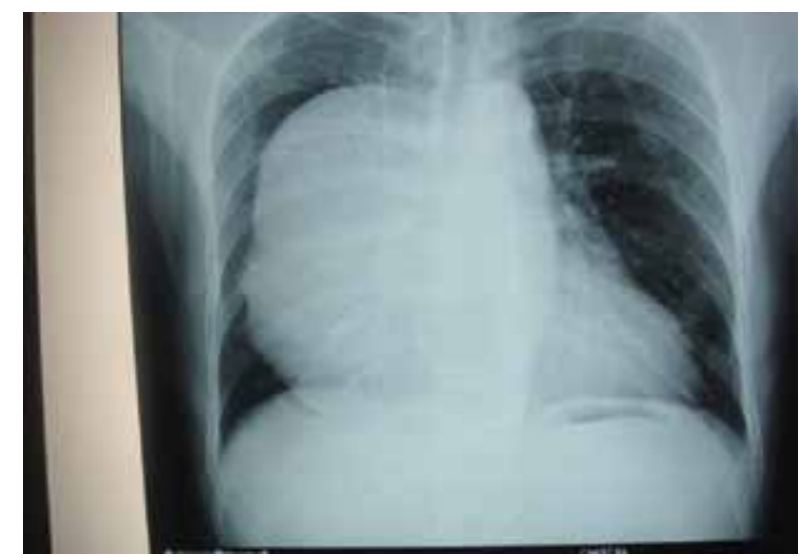

Fig. 6: Spindle cell thymoma

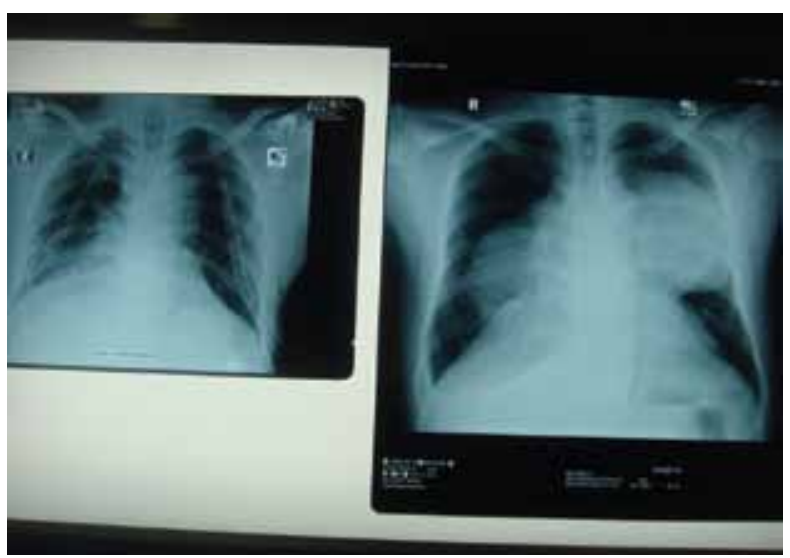

Fig. 8: Preoperative (right)and postoperative (left) $x$-ray of huge Teratoma (mature cystic teratoma) 


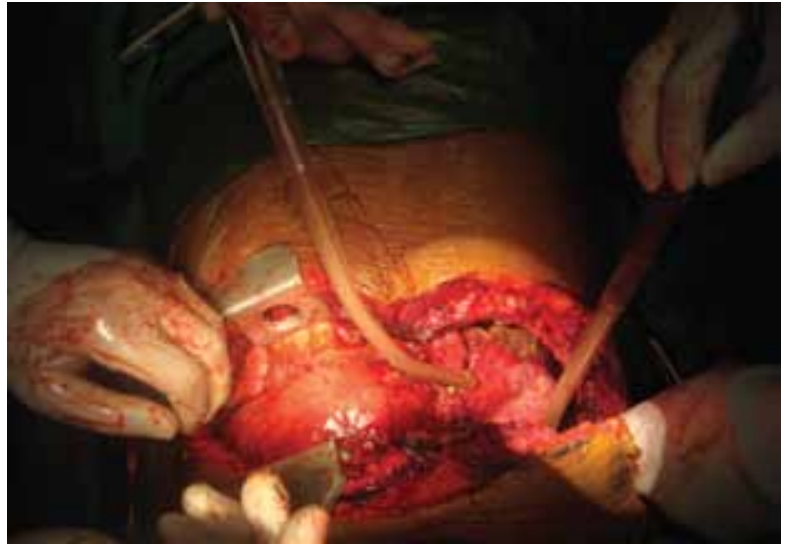

Fig. 9: Mass involving nearly whole thoracic cavity-- mature cystic teratoma

lymphoma. Masses of the middle mediastinum are typically congenital cysts, including foregut and pericardial cysts, while those that arise in the posterior mediastinum are often neurogenic tumors. The clinical sequelae of mediastinal masses can range from being asymptomatic to producing symptoms of cough, chest pain, and dyspnea $^{2}$. In our 39 patients' locations of the tumour and presentation are same type.

If left untreated, mediastinal tumors can cause serious health complications. As tumors grow, they will push against surrounding organs and tissues. Tumors that invade the heart or the vessels of the heart can cause death.

Tumors may also invade the spinal column. This may result in compression of the spinal cord.

The location of tumors within the mediastinum varies according to the age of the patient. In children, tumors are commonly found in the posterior mediastinum. These mediastinal tumors often begin in the nerves and are typically benign. In adults, most mediastinal tumors occur in the anterior mediastinum and are

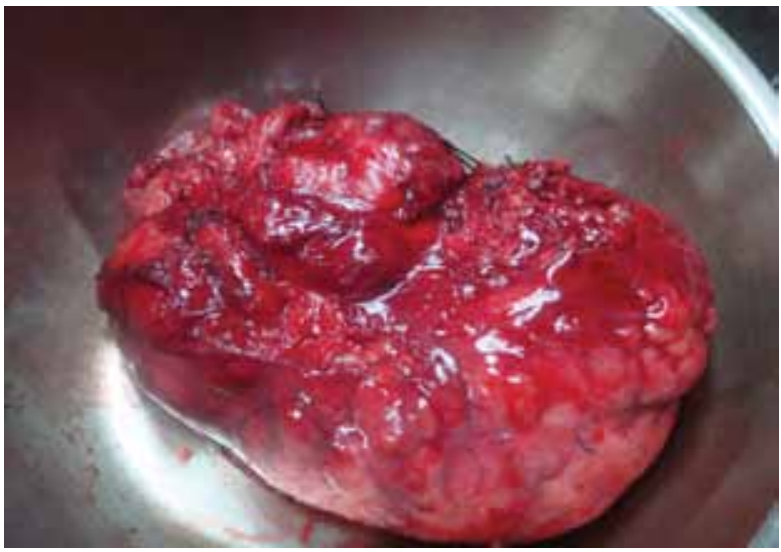

Fig. 10: Tumour about 10 inches sized spindle cell thymoma

generally malignant lymphomas or thymomas. ${ }^{4}$ Almost $40 \%$ of people who have mediastinal tumors experience no symptoms. Most of the growths are often discovered on a chest $\mathrm{x}$-ray that is performed for another reason. When symptoms are present they are often a result of the compression of surrounding structures, such as the spinal cord, heart or the pericardium. that are invading the area surrounding the mediastinum are most likely to be candidates for mediastinal surgery.

Mediastinal tumors may reach large size before becoming symptomatic. Complete surgical excision (including adjacent invaded organs) mainly by open technique should be the rule for these patients as there is survival benefit. ${ }^{6}$ Complete excisions was accomplished in all patients with benign lesions. Malignant lesions were usually partially resectable and carried a poor prognosis. $^{7} 2$ patients in our study had poor outcome due to malignant mediastinal mass (fibrosarcoma and liposarcoma). 


\section{Conclusion}

Surgical management done by cardiothoracic surgeon gives good result with good long term survival benefit. Pulmonologist and cardiothoracic anaesthetist role is also vital for good outcome.

\section{References}

1. Giron J, Fajadet P, Sans N, Jarlaud T, Verhnet H, GalyFourcade D, et al. Diagnostic approach to mediastinal masses. Eur J Radiol, 1998; 27(1):21-42.

2. Duwe BV, Sterman DH, Musani AI . Tumors of the mediastinum, Chest, 2005; 128(4):2893-909.

3. Ganesan P, Kapoor A, Bajpai J, Agarwal S, Thulkar S, Kumar L. Mediastinal Masses- the Bad, the Ugly and the Unusual! Indian J Med Paediatr Oncol, 2007; 28(3):11-16. 4. Mullen B, Richardson JD. Primary Anterior Mediastinal Tumors in Children and Adults. The, 1986; 42( 3):338-45

5. Massie RJ, Van A PP, Mellis CM. A review of open biopsy for mediastinal masses. J, 1997; 33(3):230-3.

6. Abdel RAR, Sedera MA, Mourad IA, Aziz SA, Saber TK, Alsakary MA. Posterior mediastinal tumors: outcome of surgery. J Egypt Natl Canc Inst, 2005; 17(1):1-8.

7. Al-Naaman YD, Al-Ani MS, Al-Omeri MM. Primary mediastinal tumours. Thorax, 1974; 29(4):475-81. 